\title{
Alginate Based Film Incorporated With Eugenol And/Or Limonene: Antimicrobial Activity Against Salmonella spp and Pseudomonas spp
}

\author{
Maria Crystina Igarashi (I), Mariza Landgraf (I) \\ (I) USP - Universidade de São Paulo (Av. Prof. Lineu Prestes, 580 Cep: 05508-000 São Paulo, \\ $\mathrm{SP})$
}

\section{Resumo}

The incorporation of antimicrobial substances in package films aims at reducing food microbial contamination. Among them, the phenolic compounds extract from plants have received special attention being natural and attending consumer demand. The aims of this research were to evaluate the sensitivity of Salmonella spp (S. Enteritidis ATCC 13076, S. Give, S. Enteritidis) and Pseudomonas spp (P. aeruginosa ATCC 27853, P. fluorescens, P. putida) to eugenol and/or limonene incorporated into the alginate based edible film. Alginate based films were prepared by mixing glycerin (2\%) in distilled water previously to the eugenol and/or limonene addition. Eugenol and limonene concentrations were incorporated based on the central composite rotational design $2^{2}$ with three center points. The variables were the concentration of eugenol ( 0 to $0.4 \%)$ and limonene ( 0 to $0.8 \%$ ). Sodium alginate (3\%) was then added to the solution and $\mathrm{CaCl}^{2}$ was used as a crosslinking step. The film-forming solution was then dried. After, a second crosslinking step was performed. The in vitro antimicrobial activity was carried out by the agar diffusion method using $5 \mathrm{~mm}$ diameter films placed on Mueller Hinton agar plates, which had been previously spiked with $10^{6}$ CFU.mL ${ }^{-1}$ of each of strains of Salmonella spp and Pseudomonas spp. After incubation, the zone of inhibition was measured $\left(\mathrm{mm}^{2}\right)$. Experimental data were analyzed using the software Statistica 10.0. The incorporation of eugenol $(0.2 \%)$ and limonene $(0.2 \%)$ into the alginate based film was the most effective combination against all the Pseudomonas 
spp isolates with inhibition zones ranging from $58.5 \pm 1.7 \mathrm{~mm}^{2}$ to $64.3 \pm 2.2$ $\mathrm{mm}^{2}$. For Salmonella spp none of the combinations of eugenol and limonene were as effective as the incorporation of these compounds alone with zones of inhibiton of $47.3 \pm 1.4 \mathrm{~mm}^{2}$ for eugenol $(0.4 \%)$ and $48.2 \pm 2.1$ $\mathrm{mm}^{2}$ for limonene $(0.8 \%)$. Besides being an alternative to replace nonrecyclable packaging materials, the alginate based films incorporated with eugenol and limonene plays an important role in antimicrobial packaging area. The results showed that the combination of lower concentrations of eugenol and limonene was as effective as the individual minimum inhibitory concentration for these compounds confirming the existence of synergism against Pseudomonas spp but not for Salmonella spp.

Palavras-Chave: Salmonella spp, Pseudomonas spp, eugenol, limonene, alginate based film

Agência de Fomento: FAPESP (2012/01726-2), CAPES 